\title{
ENTRE REMINISCENCE ET DIVAGATION: LES « JEUX » DE LA MÉMOIRE
}

Zeineb Ghedhahem, Université de Tunis, zeineb_3112@yahoo.fr

10.31902/fll.29.2019.10

UDK 821.133.1(64).09-31

Résumé : Selon Proust, l'écriture serait un acte de mémoire. Les écrivains pensent, s'expriment et écrivent parce que d'autres l'ont fait avant eux. D'une certaine manière, tout est réécriture et l'intertextualité est propre à tout texte. Les textes nouveaux s'emparent des textes précédents de multiples manières, la littérature multiplie ses appuis sur une « intertextualité » qui n'est autre que l'exploitation d'un fond mémoriel, d'une « mémoire de la littérature». Dans cette étude, nous nous intéresserons à la " mémoire de la littérature », autrement dit à une mémoire qui a pour objet la littérature et qui n'est pas le sujet de celle-ci dans certains romans « autobiographiques » d'Alain Mabanckou. Nous analyserons son œuvre qui manifeste d'une manière particulièrement saillante la mobilisation de la mémoire littéraire dans la construction du récit. Le texte fait écho à de nombreuses sources de la littérature mondiale qui font de la mémoire un véritable agent structurant du récit. Nous démontrerons que ces références à l'intérieur du texte sont sujettes à l'ajustement et à la modification, ce qui est aussi en fin de compte le procédé de tout acte de mémoire.

Mots-clés : littérature francophone, intertextualité, réécriture, mémoire, intertextualité.

Dans son ouvrage Proust, la mémoire et la littérature, Compagnon affirme que (Compagnon, 10) "la littérature elle-même se souvient de la littérature, elle est à la fois l'objet et le sujet de la mémoire ». Ainsi le recours au texte du précurseur montre que la création littéraire ne peut se faire sans le recours au travail de l'autre. Présentée ainsi, l'écriture serait donc un acte de mémoire.

Les écrivains pensent, s'expriment et écrivent parce que d'autres l'ont fait avant eux. D'une certaine manière, tout est réécriture et la " transtextualité ", c'est-à-dire " tout ce qui met [le texte] en relation, manifeste ou secrète, avec d'autres textes ", selon la formule de Gérard Genette, est propre à tout texte. Les textes nouveaux s'emparent des textes précédents de multiples manières, la littérature multiplie ses appuis sur une " intertextualité » qui n'est autre que l'exploitation d'un fond mémoriel, d’une " mémoire de la littérature ».

Ces emprunts appellent une vision nouvelle, une interprétation originale. Or le choix des morceaux porte-t-il une originalité en soi ? Porte-t-il la marque personnelle de l'auteur ? Quelles fonctions pourrait-on attribuer à ces mots d'autrui que Laureano Bonnet qualifie à juste titre de (Genette, 7) « cicatrices 
textuelles ", qui constituent une sorte de greffon et perdent leur statut de corps étrangers à mesure qu'elles sont absorbées par le texte qui leur fait subir des variations?

Dans cet article, nous intéresserons à la " mémoire de la littérature ", autrement dit à une mémoire qui a pour objet la littérature et qui n'est pas le sujet de celle-ci dans certains romans d'Alain Mabanckou. Nous analyserons son œuvre qui manifeste d'une manière particulièrement saillante la mobilisation de la mémoire littéraire dans la construction du récit. Le texte fait écho à de nombreuses sources de la littérature mondiale qui font de la mémoire un véritable agent structurant du récit. Nous démontrerons que ces références à l'intérieur d'un texte sont sujettes à l'ajustement et à la modification, ce qui est aussi en fin de compte le procédé de tout acte de mémoire.

Quand on envisage d'étudier l'intertextualité dans un roman quelconque, on doit s'atteler à détecter ses différentes marques, de la plus évidente à la plus subreptice. L'analyse des relations intertextuelles se doit d'être affinée, subtile, et ceci peut se réaliser par la mise en valeur des échos verbaux, thématiques, structuraux, etc., communs entre les différents genres et auteurs. Dans ce cas, la question est de définir si le parallèle ou l'allusion à tel ou tel autre auteur est délibérée ou non. Quelle que soit la réponse, nous sommes amenés à nous interroger sur sa fonction spécifique dans le discours romanesque, car l'auteur n'est pas un imitateur ou un copiste, c'est un interlocuteur qui établit un dialogue avec ses lectures, qu'il interprète à sa façon. L'intertextualité suppose donc une altérité constitutive de tout texte, comme le souligne M. Bakhtine, pour qui tous les mots de la langue sont habités par la voix d'autrui et que chaque mot est (Bakhtine, 331) " un drame à trois personnages ».

Le romancier congolais Alain Mabanckou affirme (Mabanckou, 197): " j'écris par rapport à ce que les géants du monde ont couché sur le papier". A la lecture de certains de ses romans, nous remarquons que les références à la littérature foisonnent. L'intertextualité à l'œuvre dans tous ses textes prouve que la mémoire littéraire se diffuse selon des modalités très contrastées et variables aussi bien idéologiquement qu'esthétiquement. En tant que mémoire de la littérature, l'intertextualité y est présente, de par ses différentes variantes : citation, allusion, parodie et pastiche, comme un challenge pour appréhender la littérature.

Cette mémoire littéraire est toutefois remaniée, interprétée et donc rénovée. L'écriture romanesque d'Alain Mabanckou serait-elle étroitement liée à la transmission d'une mémoire de la littérature ? Est-il possible de distinguer une poétique de la mémoire littéraire dans son œuvre?

Le texte de Mabanckou fait écho à de nombreuses sources de la littérature mondiale. Ce romancier recourt à un usage pléthorique de références intertextuelles ce qui est de toute évidence un élément principal dans sa créa- 
tion littéraire. Cela est vrai pour une majeure partie de sa production, mais nous souhaitons ici interroger trois de ses romans Black Bazar, Verre Cassé et Mémoires de porc-épic, qui manifestent d'une manière particulièrement saillante la mobilisation de la mémoire littéraire dans la construction du récit.

Dès les premières lignes, ces trois romans s'inscrivent dans une relation d'hypertextualité avec d'autres textes et surtout d'autres genres relevant aussi bien du mode d'expression oral qu'écrit. Alain Mabanckou fait partie de cette génération d'écrivains qui a décidé d'investir différents genres de textes et de discours entrainant par là même l'éclatement des frontières génériques. La création littéraire qui en découle est systématiquement modelée par la dynamique de la transgénéricité et de l'intertextualité.

Ce qui frappe à la lecture de Verre Cassé, Mémoires de porc-épic et Black Bazar, c'est le tourbillon de références littéraires, musicales, picturales, cinématographiques qui les traversent, toutes en provenance de différentes aires culturelles, créant, ce que l'écrivain franco-djiboutien Abdourahman A. Waberi appelle, (Waberi, 88) non seulement des "ponts ", des " passerelles ", mais de véritables " arborescences". Nous avons relevé pas moins de 200 références, dont certaines reviennent plusieurs fois dans les trois romans cités, à des auteurs européens, anglais, français avec une place privilégiée pour Hugo et La Fontaine. Mais des liens sont aussi tissés avec le Maghreb, l'Algérie plus précisément avec Mohamed Dib, Rachid Boudjedra, la Tunisie avec Albert Memmi. Nous rencontrons au fil de la lecture, les martiniquais Aimé Césaire et Édouard Glissant ainsi que la guadeloupéenne Simone Schwarz-Bart.

Dans ses productions littéraires, les proverbes, les dictons, les fables les clins d'œil littéraires et autres allusions jouent un rôle important. Ils circulent librement, résonnent entre eux, s'écrivent les uns à partir des autres et parfois même contre les autres. C'est là le processus de la création romanesque qui se révèle plutôt être chez Mabanckou une combinatoire des genres et d'écriture polyphonique.

"Les gens de ce pays n'avaient pas le sens de la conservation de la mémoire " (Mabanckou, 11). C'est sur ces mots que s'ouvre Verre Cassé, d'Alain Mabanckou. Verre Cassé est investi d'une mission par L’Escargot Entêté, le propriétaire du bar. Ce dernier porte le nom de L'Escargot Entêté qui est aussi le titre d'un roman de Rachid Boudjedra. Dans une interview accordée à Rachid Mokhtari, Le romancier congolais l'admet : « Oui, c'est totalement un clin d'œil au roman de Rachid Boudjedra. Je pense que Boudjedra a donné l'âge adulte à notre roman, au roman d'expression francophone ". Cette mission consiste à écrire dans un cahier la vie chaotique et sans solution d'une poignée de vaincus de la vie, afin de conserver une trace de la vie de son institution.-

Pourtant pour écrire, Verre Cassé fait appel à sa mémoire. Il cite à tour de rôle, des phrases célèbres, des titres de romans, chansons, films et séries télévi- 
sées. Il écrit à partir d'une mosaïque de citations éparses rejoignant de la sorte Balzac qui affirme que (Balzac, 5) « la littérature se sert du procédé qu'emploie la peinture qui, pour faire une belle figure, prend les mains de tel modèle, les pieds de tel autre, la poitrine de celui-ci, les épaules de celui-là ", le travail de Verre Cassé consisterait donc à « donner la vie à ces membres choisis et à [la] rendre probable " (Balzac, 367). Dans Black Bazar, le héros affirme même qu' " un écrivain est un artiste, c'est un peintre des mots... " (Mabanckou, 250).

À bien scruter les propos des différents personnages, on devine sans peine les principes prônés par l'auteur. D'une page à une autre, le lecteur finit par découvrir les goûts et les prédilections de l'auteur en matière de lecture. Ainsi dans son autre roman, African Psycho, nous pouvons lire : « Je ne vais pas me mettre à citer ceux qui m'ont fasciné. Toutefois, je peux dire que ceux que j'ai lus m'ont permis de voyager, d'aller loin, de voir d'autres horizons» (Mabanckou, 32). Et un peu plus loin : " Je me suis lancé, moi aussi, dans la lecture de ce que les gens appellent la grande littérature » (Mabanckou, 32).

Le nom même de Verre Cassé lui vient d'un souvenir de l'auteur : avant de désigner le personnage éponyme du roman de Mabanckou, Verre Cassé est le titre d'une chanson de Lutumba Simaro, poète et chanteur Congolais, dont la poésie s'entend dans Black Bazar, Verre Cassé et ses autres romans. La chanson évoque une vie amoureuse brisée et célèbre le surgissement du souvenir réparateur.

Au cours d'une interview accordée à Rachid Mokhtari, Alain Mabanckou déclare :

Donc, je pense que mon roman "Verre cassé " est aussi une fête de littérature : "L'Escargot entêté ", "Le fleuve détourné ", "La nuit sacrée ", "Voyage au bout de la nuit "... J'ai essayé, à travers ces clins d'œil, de former une narration qui soit l'ivresse des livres. Mes livres sont constitués de personnages qui boivent beaucoup, mais ce qu'on oublie souvent, de préciser, c'est qu'ils boivent aussi les livres. La plus grande ivresse est celle des livres. Celui qui boit les livres a une ivresse intarissable. Et de ce fait, il devient en quelque sorte celui qui préserve la mémoire.

D'une certaine manière, l'auteur tient à ce que le cahier rédigé par Verre Cassé s'inscrive d'emblée dans un rapport de filiation avec les textes qui le précèdent. Le narrateur est en état d'addiction par rapport à la littérature, c'est un boulimique de la lecture, son cahier se transforme en une sorte de Bibliothèque réunissant des titres d'œuvres de tous horizons et s'il est mémoire des vies vécues par les clients qui ont échoué au Crédit a voyagé, il se fait aussi mémoire des livres lus, des films vus et des chansons entendues par l'auteur et ses personnages. 
Truffé de références littéraires, Black Bazar forme un long dialogue entre les écrivains et les œuvres. "C'est un roman qui magnifie la littérature et rend hommage aux écrivains que j'ai aimés ", explique Mabanckou. Roger Le FrancoIvoirien, dans l'une de ses interminables interventions, justifie le recours incessant à l'intertextualité en vociférant : " Tout a déjà été écrit ici-bas, Fessologue ! Tout ! Et moi j'ai lu tous les grands livres du monde ! » (Mabanckou, 20).

Mabanckou parvient à faire de son roman une œuvre qui se caractérise par l'amalgame des morceaux de textes choisis, sorte de canevas textuel constitué de fragments en apparence décousus. C'est dans le travail d'assemblage et de collage que résident la création et l'originalité comme en témoigne cet extrait de Verre Cassé :

Je me souviens toujours de ma première traversée d'un pays d'Afrique, je goûtais aux fruits si doux de l'arbre à pain, j'habitais dans une chambre de l'hôtel la vie et demie qui n'existe plus de nos jours et où, chaque soir, entre Jazz et vin de palme, mon père aurait exulté de joie, et je me réchauffais au feu des origines, pourtant il fallait aussitôt repartir, ne pas s'enfermer dans la chaleur de la terre natale, sillonner le reste du continent pour écouter les élégies majeures, le chant d'ombres il fallait traverser les villes cruelles dans l'espoir de rencontrer un dernier survivant de la caravane (Mabanckou, 171).

Grâce à un trait d'esprit, à connotation humoristique, Verre cassé réussit à truffer les pages de certains titres de romans africains dans une sorte de calembours, pour le plus grand bonheur des férus de la littérature africaine. II reprend ces titres et convie le lecteur à une virée à travers l'Afrique. Nous devinons qu'il s'agit des œuvres de Camara Laye (L'Enfant noir), Aimé Césaire (Cahier d'un retour au pays natal), Tchikaya Utamsi (Les fruits si doux de l'arbre à pain), Sony Labou Tansi (La vie et demie), Emmanuel Dongala (Jazz et vin de palme), Léopold Sédar Senghor (Chant d'ombre), Mongo Béti (Ville cruelle).

Le style du romancier congolais est majoritairement composé de références à des textes littéraires. II partage ses lectures et crée, avec elles, son propre travail d'auteur. En tant que lecteurs, nous sommes alors intrigués par I'usage des citations, ces bribes de textes, quelquefois familiers, qui parcourent son œuvre. II recourt à une utilisation systématique des mots des autres par les citations et allusions littéraires. Cette écriture est celle de la conscience libre, de la pensée qui réanime les mots, qui leur rend la vie. Ses romans se servent de la littérature comme prétexte. Lire pour Mabanckou reviendrait à une appropriation, une réquisition du texte d'autrui ; citer serait proposer sa propre lecture, subjective, déformée et déformante, recréer du texte. Autrement dit, citer, c'est considérer la lecture comme écriture. Le recours à la citation s'inscrit dans un projet de collaboration, le texte cité, de par son intégration dans le texte second, assure à ce dernier la possibilité de se " créer » et de se développer. 
Acte de référence et acte de révérence, la citation du discours de l'Autre est une forme de reconnaissance. Par ailleurs, citer est une façon de se faire reconnaître. En rendant hommage à l'auteur du texte cité, Mabanckou s'affirme à son contact. Dans African Psycho, le narrateur dit : " Ne pouvant égaler les prouesses du Grand Maître, j'aimerais au moins être considéré comme son fils spirituel. Pour cela, j'en suis conscient, je dois encore travailler » (Mabanckou, 9). Le plus souvent, la citation est le moyen dont disposent les personnages pour exprimer une idée. Elle se présente comme une formule à laquelle ils recourent pour donner forme à leurs pensées et consolider leurs propos. Ces personnages en viennent à penser dans les termes de la littérature. L'insertion du mot d'autrui dans le texte, non seulement réunit deux voix, mais permet en outre à l'auteur de placer son récit sous l'autorité d'un autre avec lequel il établit une relation égalitaire, une sorte de lien intellectuel, ainsi qu'une parenté spirituelle.

En affichant une certaine relation avec ces romanciers notoires, l'auteur cherche à tirer profit d'un " chakra » de la connaissance, constitué à partir de diverses identités. L'auteur ne se contente pas d'une simple évocation de ces modèles mais il incite à les dépasser. Cette présence subtile dans le tissu textuel est manifeste dans cet exemple : « l'infortune d'un vieil homme qui allait pêcher en haute mer et qui devait lutter seul contre un gros poisson, ce poisson était à mon avis un double nuisible d'un pêcheur qui jalousait ce vieux et son expérience » (Mabanckou, 156).

Etant à la recherche d'une consolidation à son opinion, d'un moyen pour étayer son argumentation, l'auteur fait appel, dans certains cas, à l'autre, le convie afin d'orner son texte d'une formulation plus heureuse. Certains vers de La Fontaine sont, à ce titre, jugés dignes d'illustrer les propos du patriarche dans Mémoires de porc-épic : "fi du plaisir que la crainte peut corrompre ". Ces emprunts, non seulement établissent une connivence mais sont également appréhendés comme des arguments d'autorité.

Parfois, cette relation peut aussi traduire une certaine hostilité, rendue possible grâce au procédé de déviance, comme dans cet exemple : (Mabanckou, 65) "le rire n'a pas toujours été le propre de l'homme ". Le modèle est tout simplement modifié, subverti. Le mouvement est double : en premier lieu, l'auteur convoque et s'approprie l'emprunt pour ensuite le modifier en vue de l'adapter au contexte. C'est cette subversion qui est le gage de la créativité de l'auteur et de son pouvoir innovateur. Ces innovations linguistiques sont les pratiques invoquées pour rendre compte de ce virage décisif vers une écriture résolument moderne, voire postmoderne.

Une relation singulière s'établit entre le roman et cette " mosaïque de citations " qu'il absorbe. Laurent Jenny rend compte de cette relation en soulignant : 
[il] faut que le texte " cité » admette de renoncer en quelque sorte à sa transitivité : il ne parle plus, il est parlé. Il ne dénote plus, il est connoté. Il ne signifie plus pour son propre compte, il passe au statut de matériau (Jenny, 267).

En effet, si l'intertexte, de par sa simple présence, modifie le roman, ce dernier en fait de même et procède de surcroit à son interprétation. Quel que soit son genre et le traitement qu'il subit, l'intertexte n'est jamais anodin. II s'inscrit toujours dans une stratégie narrative et esthétique. Quelle que soit la nature de son intégration, c'est presque toujours une sollicitation, et sa reprise, indépendamment de sa forme, vise toujours à le ressusciter.

En fait, l'auteur postmoderne ne se réinvente pas : il puise plutôt dans ce qui existe déjà, récupère ce qui l'intéresse et en forme une œuvre distincte. II mélange les styles de différentes époques, les différents arts et formes d'expression, comme pour refléter la réalité actuelle, multiple et diversifiée.

De ce fait, le texte de Mabanckou ne peut être appréhendé comme un espace clos, mais comme un ensemble ouvert, se pliant à maintes interprétations du lecteur. Même s'il se présente comme un patchwork de citations, qui amène à prendre au pied de la lettre l'affirmation de J. Kristeva que (Kristeva, 145 ) "tout texte se construit comme une mosaïque de citations ", la lecture ne se contente plus du repérage de simples filiations par rapport à un texte source. En effet, la lecture intertextuelle ne se réduit pas à un simple rappel érudit des sources ou des emprunts d'un auteur, mais devient un levier, un code pour l'interprétation. Elle incite à être attentif à la lecture que l'auteur fait des mots, des textes ou des genres qu'il absorbe et détourne dans son propre texte. L'originalité et l'inventivité de notre romancier ne résident pas dans sa capacité à imiter, même s'il le revendique parfois, mais plutôt à sa capacité de mettre en place tout un processus de transformation, d'appropriation ou de détournement des textes sources et même des genres imités

Les références à une telle variété d'œuvres ou d'auteurs placent d'emblée l'œuvre dans la bibliothèque universelle. Tout au long du récit, le héros-narrateur ne cesse de faire appel à sa mémoire et à son érudition au point que Mémoires de porc-épic se situe dans la bibliothèque universelle de par la quantité phénoménale et la variété des références littéraires. Dans Verre cassé, le héros écrit à ce propos : "le nègre en question est une véritable bibliothèque ambulante, [...] il parle comme un livre ». En effet, les traces intertextuelles plus ou moins fugaces, au sens où l'entend Laurent Jenny, foisonnent.

Une des originalités du style de Mabanckou est que son écriture est allusive et qu'elle joue sur l'absence, le non-dit, le sous-entendu. C'est au lecteur de fournir l'effort du déchiffrement. De simple lecteur, il devient un complice, un co-auteur invité à compléter ce qui est esquissé afin de reconstruire le sens. Ses 
romans offrent différents parcours de lecture en fonction des lecteurs. Les propos de Julián Ríos rendent compte de manière édifiante de cette spécificité scripturale :

Il y a des livres simplement masturbateurs, la plupart, ces livres qu'on lit à la sauvette, et qu'on jette et qu'on oublie tout de suite. Mais il y a d'autres livres qui ne se livrent pas à la première lecture et qui exigent qu'on leur fasse la cour. Je pense finalement que ce sont ces livres qui valent la peine d'être lus, et qu'on n'aura jamais fini de lire (Rios, 24).

Alain Mabanckou multiplie ainsi les allusions, sans que celles-ci soient nécessairement repérées comme telles. Dans le discours du porc-épic, les allusions textuelles sont soumises à un traitement ludique, on peut même dire manipulées sur un mode ironique, ce qui aboutit parfois à des associations savoureuses comme dans l'extrait suivant :

c'est toujours Amédée qui leur racontait à plusieurs reprises l'histoire d'un gars qui se déplaçait sur un tapis volant, d'un patriarche qui créa un village appelé Macondo et dont la descendance allait sombrer dans une espèce de malédiction, naître mi-homme mi-animal, avec des groins, des queues de cochons, je suis persuadé qu'il devait s'agir aussi d'histoires de doubles nuisibles, et, autant que je m'en souvienne, il contait les aventures d'un type bizarre qui combattait tout le temps contre des moulins à vent, ou alors dans le même ordre d'idées, l'infortune d'un officier qui espérait en vain des renforts dans un camp perdu dans le désert, et que dire de ce vieux colonel qui attendait une lettre et sa pension d'ancien militaire, ce malheureux colonel qui vivait dans le dénuement avec son épouse malade et leur coq de combat sur lequel ils fondaient leurs espoirs, ce coq était leur seule lueur, cet animal devait être un double plutôt pacifique, donc je n'insiste pas (Mabanckou, 143).

Une pléthore d'allusions littéraires innerve le discours de Ngoumba. Caractérisé par son encodage extratextuel, ce type d'allusion manifeste ainsi un mode de fonctionnement dialogique et appelle de la part du lecteur un effort de déchiffrage qui tienne compte de cette dynamique. Si la citation, de par son insertion dans le texte, révèle d'emblée son hétérogénéité énonciative, l'allusion procède autrement. En effet, cette dernière, de par son côté ludique voire énigmatique, procède d'un dialogisme plus contenu, plus discret.

Les traces subtiles qui se laissent entrevoir, requièrent l'identification des textes sources. Nous retrouvons Aladin sur son tapis assistant aux combats surréalistes de Don Quichotte, alors qu'une illustre famille est condamnée à cent ans de solitude par la prophétie d'un gitan. Tandis que le vieux colonel de Faulk- 
ner attend, encore et toujours, sa pension de retraité tout en veillant à la survie de son coq de combat. Toutes ces œuvres sont considérées comme des pièces maîtresses de la littérature universelle.

Les romans de Faulkner, Gabriel Marquez, Cervantès entrent en résonnance pour raconter des histoires cruelles et dérisoires et parfois invraisemblables, des histoires faisant la part belle aux mythes qui engendrent les hommes qui à leur tour engendrent les mythes. Tout porte à croire qu'il y a une volonté de la part du héros-narrateur de produire une œuvre universelle dans laquelle résonneraient les voix de Gabriel Garcia Marquez, de Cervantès, d’Hemingway, de Faulkner et tant d'autres encore. L'auteur, dans un désir inavoué d'impressionner le lecteur, laisse apparaitre sa vaste culture. Sa mémoire personnelle est impressionnante.

L'intertextualité, et par ricochet l'allusion, grâce à la dextérité du hérosnarrateur, devient féconde. L'hypotexte soumis aux différents procédés ludiques et comiques auxquels le contraint l'auteur, se joue de tout, détruit des mythes pour en créer de nouveaux, désacralise le sérieux en y greffant du trivial. Le dessein subversif est le principe même de la production et de la création, son " dada ». L'allusion littéraire apparait comme marque d'une érudition et prétexte que le roman s'attache à détourner, tel cet exemple à relent shakespearien de Verre Cassé : « mon cœur balançait vraiment, la brousse ou pas la brousse, telle était la question » (Mabanckou, 179).

En tant que configuration discursive particulière, l'allusion enchâsse, dans son propre énoncé, un autre énoncé connu et reconnaissable, qui peut être entier, détourné ou non. Dans Verre Cassé, nous lisons :

Le président-général des armées a dit qu'on devrait traquer ces félons, ces marionnettes, ces hypocrites, il les a carrément traités de tartuffes, de malades imaginaires, de misanthropes, de paysans parvenus (Mabanckou, 32).

L'allusion intertextuelle renvoie à un hors-texte, à un intertexte externe identifiable, tourné vers le déjà-dit, parfois vers "le multiplement-déjà-dit ". Dans cet exemple, l'allusion relève plutôt d'un montage, un fondu-enchainé des titres des œuvres de Molière et de Marivaux.

Au fil des pages, ce processus s'affine de plus en plus dans la mesure où les intertextes s'intègrent parfaitement dans la phrase et font sens. C'est le cas par exemple de ce passage tiré de Verre cassé:

« Je suis allé errer vers le quartier Rex, à l'ombre des jeunes filles en fleurs, il n'y a eu aucune jeune fille en fleur qui a voulu de moi, il n'y a eu aucune jeune fille en fleur qui a voulu que je tire un petit coup vite fait » (Mabanckou, 54). 
Tous ces phénomènes de citations, de paraphrase, d'allusions se déroulent sous forme de jeux « taquins » dont l'objet est le labeur sérieux associé communément à l'écriture.

L'accumulation d'allusions ne correspond pas à une singularité. Les allusions fonctionnent la plupart du temps en corrélation les unes avec les autres. Elles constituent un réseau interactif qui les fait se répondre, s'opposer et s'inclure. Ce mode de fonctionnement est assez clair dans les extraits proposés.

Les références à des auteurs, à des œuvres foisonnent dans le texte de Mabanckou, ce qui fait dire à Mathieu Csernel :

Alain Mabanckou glisse toutes les deux lignes une référence littéraire. [...] Discrètes, elles ne ralentissent jamais le récit. Mais lorsqu'on en trouve une c'est un petit moment de plaisir supplémentaire en repensant au livre auquel il est fait allusion et qu'on a la joie de connaitre.

Heureuse ou malheureuse, la réminiscence est l'ombre de ce souvenir qui inspire et influence la création, l'écriture. L'emprunt, le plagiat ainsi réalisé surgit d'une mémoire profonde, lointaine, des tréfonds de l'être. Réminiscence de l'enfance, de la jeunesse; réminiscence de lecture, souvenir où domine plus ou moins l'élément affectif. L'écriture devient conservation des traces, mémoire, mais une mémoire pillarde et blagueuse. Selon André Lamontagne, la réminiscence résulte du fait qu'un personnage est mis « en situation d'énonciation par une instance narrative supérieure, situation somme toute analogue à celle où un personnage est posé comme lecteur » (Lamontagne, 42).

« Les multiples brindilles » qui constituent le « nid littéraire » d'Alain Mabanckou se distinguent par la diversité des origines des auteurs cités. Du dialogue entre les textes et les cultures, on passe donc à l'universel. Dans ce nouvel espace qu'est le roman, Alain Mabanckou s'octroie, avec jubilation, le droit de se livrer à des frasques littéraires qui libèrent la parole. L'écriture ironique établit alors le dialogue entre les littératures et les cultures.

Pour Verre cassé, être intellectuel rime avec liberté et transgression (Mabanckou, 154) : «chacun cultive son jardin comme il peut » dira-t-il. Le scribe se défait d'un certain conformisme inhibiteur et affiche l'image d'un intellectuel plutôt retors doublé d'une attitude anticonformiste et un élan interculturel inégalé. A travers cette intertextualité dialogique qui innerve ces textes transparait le désir d'instaurer une éthique de l'interculturel, seule garante d'un dialogue constructif entre les cultures et qui trouve son expression pragmatique à travers le concept de Littérature-monde. Ce dernier a été annoncé dans un manifeste dont Mabanckou est l'un des signataires et des fervents défenseurs.

Alain Mabanckou se place dans une posture de dialogue manifeste avec la tradition, c'est un auteur qui expérimente, et qui, par ses expérimentations, enrichit et renouvelle le genre romanesque. Dans sa pratique d'écriture ac- 
tuelle, l'intertextualité semble être au centre de la conception de la création romanesque. L'intertextualité dans les trois romans est omniprésente, polymorphe et complexe. Ses différentes manifestations obligent le lecteur à faire face à maintes difficultés et contraintes liées à la coprésence de textes littéraires d'auteurs différents, du même auteur et d'autocitations. Les parcours intertextuels varient selon le lecteur. Ils sont tributaires de ses compétences et de son érudition, ce qui le contraint à abandonner son statut de lecteur-passif pour endosser celui de lecteur-explorateur.

\section{Références bibliographiques}

Authier-Revuz, Jacqueline. Ces mots qui ne vont pas de soi. Boucles réflexives et non-coïncidences du dire. Paris : Larousse, 1995. Imprimé.

Bakhtine, Mikhail. Esthétique de la création verbale. Paris : Gallimard, 1984. Imprimé.

Balzac, Honoré. Préface du Cabinet des Antiques .L'œuvre de Balzac XV. Paris : Michel Lévy Frères Editeurs, 1839. Imprimé.

Compagnon, Antoine (Dir). Proust, la mémoire et la littérature. Paris : Odile Jacob, coll. «Collège de France », 2009. Imprimé.

Divertissement/livres/critiques/2009. Web. 18-05-2016.

Freealgerie.com/debat-du-jour/305-alain-mabanckou-humour-de-la-negrailleparisienne.Web.18-05-2016.

Genette, Gérard. Palimpsestes, la littérature au second degré. Paris : Seuil, 1982. Imprimé.

Jenny, Laurent. « La Stratégie de la Forme », in Poétique, n²7 (1976) : 259- 267. Imprimé

Kristeva, Julia. La Révolution du langage poétique. Paris : Seuil, 1974. Imprimé.

Lamontagne, Alain. Les mots des autres : la poétique intertextuelle des œuvres romanesques d'Hubert Aquin. Québec : PUL, 1992. Imprimé.

Mabanckou, Alain. African Psycho. Paris : Ed. Le Serpent à plumes, 2003. Imprimé.

Mabanckou, Alain. Verre Cassé. Paris : Seuil, 2005. Imprimé.

Mabanckou, Alain. Mémoires de porc-épic. Paris : Seuil ; 2006. Imprimé.

Mabanckou, Alain. Black Bazar. Paris : Seuil, 2009. Imprimé.

Revue critique de fixion française contemporaine. Web. 19-05-2016.

Ríos, Julian. Larva, un jeu de patience, in Magazine littéraire, $\mathrm{n}^{\circ} 170$ (mars 1980). Imprimé.

Waberi, Abdourahman Ali. Balbala. Paris : Ed. Serpent à plumes, Coll. Fiction, Domaine Français, 1998. Imprimé. 


\section{BETWEEN REMINISCENCE AND WANDERING: THE “GAMES" OF MEMORY}

According to Proust, writing would be an act of memory. Writers think, speak and write because others have done it before them. In a way, everything is rewritten and intertextuality is unique to any text. The new texts taking hold of the preceding texts in many ways, the literature multiplies its support on an "intertextuality" which is none other than the exploitation of a memorial background, a "memory of literature". In this study, we will be interested in the "memory of literature", in other words, in a memoir which has as its object literature and which is not the subject of literature in certain "autobiographical" novels by Alain Mabanckou. We will analyze his work which manifests in a particularly salient way the mobilization of the literary memory in the construction of the narrative. The text echoes many sources of world literature that make memory a true structuring agent of the narrative. We will demonstrate that these references within the text are subject to adjustment and modification, which is also ultimately the process of any act of memory.

Keywords: French literature, intertextuality, rewriting, memory. 